Article

\title{
Normalized Expected Utility-Entropy Measure of Risk
}

\section{Jiping Yang* and Wanhua Qiu}

School of Economics and Management, Beihang University, Beijing 100191, China; E-Mail: 01661@buaa.edu.cn

* Author to whom correspondence should be addressed. E-Mail: yangjp@buaa.edu.cn; Tel.: +86-10-82339895; Fax: +86-10-82317832.

Received: 5 May 2014; in revised form: 24 June 2014 / Accepted: 25 June 2014 /

Published: 30 June 2014

\begin{abstract}
Yang and Qiu proposed an expected utility-entropy (EU-E) measure of risk, which reflects an individual's intuitive attitude toward risk. Luce et al. have derived the numerical representations under behavioral axioms about preference orderings among gambles and their joint receipt, which further demonstrates the reasonability of the EU-E decision model as a normative one. In the paper, combining normalized expected utility and entropy together, we improve the EU-E measure of risk and decision model, and then propose the normalized EU-E measure of risk and decision model. The normalized EU-E measure of risk has some normative properties under certain conditions. Moreover, the normalized EU-E decision model can be a proper descriptive model to some extent. Using this model, two cases of common ratio effect and common consequence effect, which are the examples of certainty effects, can be explained in an intuitive way.
\end{abstract}

Keywords: risk; normalized expected utility; normalized entropy; certainty effect; common ratio effect; common consequence effect

\section{Introduction}

The measure of risk, and furthermore decision making under risk have always been important issues in the field of decision sciences, finance, economics and psychology, etc. The dominant decision analysis under risk is the expected utility theory ([1]) due to its role of being either a normative model of rational choice or a descriptive model of economic behaviors [2]. Since the challenge of its descriptive power for risky choices arose, various measures of risk for risky actions and decision 
analysis models have been established to provide additional insights into risky decision problems. Some of these researches have been performed by Kahneman and Tversky [2], Levy [3], Dyer and Jia [4], Jia et al. [5], Dionisio et al. [6], Marley and Luce [7], Ng et al. [8], Luce et al. [9-11], etc.

Yang and Qiu [12] proposed the expected utility-entropy (EU-E) measure of risk and a decision making model based on expected utility and entropy of an action involving risk. The EU-E measure of risk is based on the general decision-making model under risk [12], in which different actions may correspond to different states of nature and each state of nature may have its own distribution. For a general decision model $G=(\Theta, A, u), \Theta=\{\theta\}$ is the state space, $A=\{a\}$ is the action space, $u(X)$ is the decision maker's utility function, while $X=X(a, \theta)$ is the payoff function defined on $A \times \Theta$.

Specifically, when both action and state space are finite, $A=\left\{a_{1}, a_{2}, \cdots, a_{m}\right\}$, the state $\theta_{i}$ corresponding to $a_{i}$ has $n_{i}$ outcomes $\theta_{i 1}, \theta_{i 2}, \cdots, \theta_{i n_{i}}$, the payoff is $X=X\left(a_{i}, \theta_{i j}\right)=x_{i j}$ when taking action $a_{i}$ while state $\theta_{i j}$ occurs, and the probability distribution of $\theta_{i}$ is $\left\{p_{i j}\right\}, p_{i j}=P\left\{X=x_{i j}\right\}$ is the probability that outcome $x_{i j}$ occurs $\left(i=1,2, \cdots, m ; j=1,2, \cdots, n_{i}\right)$. Then, the general decision making model can be tabled [12].

For the action $a_{i}$, it is also denoted by a combination of outcomes and corresponding probabilities in the following matrix or vector form used in [7-11]:

$$
a_{i}=\left(\begin{array}{cccc}
x_{i 1} & x_{i 2} & \cdots & x_{i, n_{i}} \\
p_{i 1} & p_{i 2} & \cdots & p_{i, n_{i}}
\end{array}\right) \text {, or } a_{i}=\left(x_{i 1}, p_{i 1} ; x_{i 2}, p_{i 2} ; \cdots ; x_{i, n_{i}}, p_{i, n_{i}}\right) .
$$

In our insight of the notion of risk in decision analysis, there are two main factors that determine the decision maker's choice of action: the uncertainty of outcomes resulting from uncertainty of occurrence of state and decision maker's expected utility when taking a certain action. Based on this insight of risk, the EU-E measure of risk of action $a$ is defined as follows [12]:

$$
R(a)=\lambda H_{a}(\theta)-(1-\lambda) E[u(X(a, \theta))] / \max _{a \in A}\{E[u(X(a, \theta))]\},
$$

where $0 \leq \lambda \leq 1$ is a constant, $H_{a}(\theta)$ denotes entropy of the distribution of its corresponding state. Suppose the utility function is nonnegative and $\max _{a \in A}\{E[u(X(a, \theta))]\}$ exists.

The constant $\lambda$ reflects a tradeoff between a decision maker's subjective expected utility of an action and objective uncertainty of its corresponding states. In EU-E measure of risk, the expected utility reflects the decision maker's subjective preference; the entropy measures the objective uncertainty of its corresponding states. The EU-E measure of risky action effectively incorporates the decision maker's subjective preference and the objective uncertainty regarding the states of nature by the risk tradeoff factor. This measure of risky action is the weighted linear average of the expected utility and entropy. If all actions have an equal expected utility, then the risk ordering is determined by the entropy component. This result was derived by Luce [13]. If all states of nature have the same distribution, then the entropy of the states is the same for every state of nature corresponding to each action; in this case the risk ordering is determined by the expected utility component.

Recently, Marley and Luce [7] presented a detailed theoretic analysis of five utility representations-subjective expected utility, rank-dependent utility, gains decomposition utility, rank weighted utility, and a configural-weighted model. Luce et al. [10] have derived the numerical representations under behavioral axioms about preference orderings among gambles and their joint 
receipt. These representations are for uncertain alternatives and consist of a subjective utility term plus a term depending upon the events and the subjective weights.

For the risky case, Luce et al. [11] specialize the results on entropy-modified representations of event based gambles to representations of probability-based gambles by assuming an implicit event structure underlying the probabilities. Under segregation and under duplex decomposition conditions, they obtain numerical representations consisting of a linear weighted utility term plus a term corresponding to information-theoretical entropies.

Under segregation assumption, Luce et al. [11] present an exact representation, which is called entropy-modified expected utility (EM-EU) [11]. Using notations in the general decision-making model, the EM-EU representation of action $a$ in Luce et al. [11] is in the following form:

$$
U(a)=E[u(a)]+A \cdot H_{a}(\theta),
$$

where, $U(a)$ is the representation of risky action $a, E[u(a)]$ is the expected utility of action $a, H_{a}(\theta)$ is the Shannon entropy of state of nature corresponding to action $a$, and $A$ is a constant.

Luce et al. [11] provide an explanation of a number of the well-known empirical paradoxes using EM-EU representation. Their results are very similar to those of Yang and Qiu [12]. This further demonstrates the reasonability of EU-E decision model in Yang and Qiu [12].

The EU-E measure of risk is a closely related non-axiomatized representation involving Shannon's entropy [9]. This measure of risk tends not to be axiomatic, not to be mathematically very general, and not to apply to uncertain alternatives [10,11]. Using the EU-E decision model, some well-known decision problems including the famous Allais paradox can be solved reasonably. Actually, this happens with an underlying assumption that the numbers of state of nature are equal, or relatively close. While the numbers of the state of nature corresponding to risky actions are far apart, both EU-E measure of risk and EM-EU representation may not be appropriate representations for risky choice.

Let us investigate the choices between the following pairs of risky actions in Table 1.

Table 1. Pairs of risky actions.

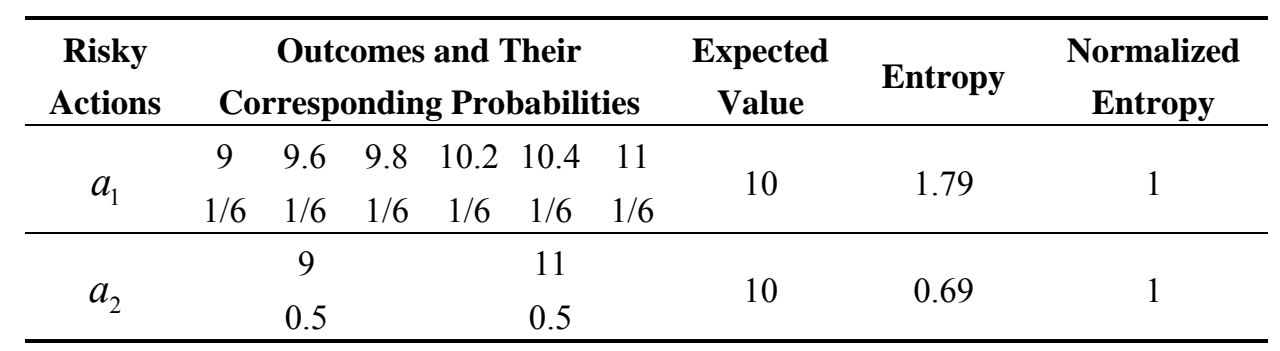

For the risky actions in Table 1, they have the same expected value. If the utility function is linear, it would be concluded that action $a_{1}$ may be much uncertain than action $a_{2}$. Using the EU-E measure of risk, the individual should choose $a_{2}$. If we use EM-EU representation for risky choices, there hold $U\left(a_{1}\right)=10+1.79 A, U\left(a_{2}\right)=10+0.69 A$, where $A$ is a constant. Thus, $a_{2} \succ a_{1} \Leftrightarrow U\left(a_{1}\right)<U\left(a_{2}\right)$ $\Leftrightarrow A<0$. But people may perceive that they have the same relative uncertainty, and $a_{1}$ may have higher expected utility and choose $a_{1}$. In this case, both EU-E and EM-EU representation may not provide the proper description for risky choice.

To deal with these kinds of risky actions, normalized entropy is a better way to measure the relative uncertainty of the risky actions with different numbers of state of nature. In addition, the value of the 
EU-E measure of risk is not the standardized one, so it is necessary to extend the EU-E measure of risk and the corresponding decision model.

In this paper, we improve the EU-E measure of risk as well as the EU-E decision model to propose a normalized EU-E measure of risk and decision model for the general decision-making model under risk. Then, we explore some properties of the normalized EU-E measure of risk. Using the normalized EU-E decision model, the certainty effect has been interpreted in a simple way. We also compare the predictions of the (normalized) EU-E and EM-EU presentations, and discuss similarities and differences among these representations. We demonstrate the reasonability of the normalized EU-E decision model as a descriptive model or a normative decision model involving risk.

\section{Normalized Expected Utility-Entropy Measure of Risk}

In this paper, we use the normalized entropy to measure the relative uncertainty of the state of nature $\theta$ of a risky action. The entropy is a measure of the amount of uncertainty in a probability distribution originally defined by Shannon [14]. When the state of nature $\theta$ corresponding to action $a$ is a discrete variable with a set of probabilities $p_{1}, p_{2}, \cdots, p_{n}$, the entropy of $\theta$ is defined as:

$$
H_{a}(\theta)=-\sum_{i=1}^{n} p_{i} \ln p_{i}
$$

The normalized entropy is defined as $N H_{a}(\theta)=\left(-\sum_{i=1}^{n} p_{i} \ln p_{i}\right) / \ln (n)$, for $n>1 ; N H_{a}(\theta)=0$, for $n=1$. The maximum uncertainty reaches when the state of nature follows the uniform distribution with $n$ outcomes, and $\ln (n)$ represents maximum uncertainty. A value $N H_{a}(\theta)=0$ implies no uncertainty (i.e., $p_{i}=1$ for some $i$ and $p_{j}=0$ for all $j \neq i$ ). Alternatively, an $N H_{a}(\theta)=1$ implies maximal uncertainty (i.e., $p_{i}=1 / n$ for all $i=1,2, \cdots, n$ ).

The normalized entropy is a measure of relative uncertainty [15]. The value of normalized entropy lies between 0 and 1 , so $N H_{a}(\theta) \in[0,1]$. This leads to standardized measures which can be compared with one another [16]. An analog measure $1-N H_{a}(\theta)$, called the information index, serves to measure the reduction in uncertainty [17].

For a risk-averse decision maker, it would be better that risky action has the smaller relative uncertainty, i.e., the smaller normalized entropy. This is consistent with notion of risk proposed by Yang and Qiu [12]. Therefore, we should take these two sides into account. People may wish to reduce uncertainty and increase expected utility of an action.

This insight of risk has motivated us to improve the EU-E measure of risk for an action in context of the general decision analysis model to the normalized EU-E measure of risk.

Definition 2.1. Given a general decision analysis model $G=(\Theta, A, U)$, action $a \in A$, state of nature $\theta \in \Theta$. Suppose the utility function $u(x)$ is mono-increasing, and $u(x) \geq 0$, we have $\max _{a \in A}\{E[u(X(a, \theta))]\}>0$. Then, the normalized EU-E measure of risk of action $a$ when taking action $a$ is defined as follows:

$$
R(a)=\left\{\begin{array}{cc}
\lambda N H_{a}(\theta)-(1-\lambda) N E(a), & \text { if } n>1 \\
-(1-\lambda) N E(a), & \text { if } n=1
\end{array},\right.
$$


where $N H_{a}(\theta)=H_{a}(\theta) / \ln (n), N E(a)=E[u(X)] / \max _{a \in A}\{E[u(X(a, \theta))]\} ; n$ is the number of actions in action space; $\lambda \in[0,1]$ is a constant, $H_{a}(\theta)$ is the entropy of the states of nature $\theta$ corresponding to action $a$; $X(a, \theta)$ denotes the outcome corresponding to state $\theta$ when taking action $a$.

In Equation (5), $N E(a)$ is the normalized expected utility of the action $a$, and $N H_{a}(\theta)$ is the normalized entropy of the state of nature $\theta$. We have $0 \leq N H, N E \leq 1$, then it holds that $-1 \leq R(a) \leq 1$.

The Definition 2.1 provides a quantified measure of an individual's intuitive perception of an action's risk. It is the weighted linear average of normalized expected utility and entropy. The definition of risk is based on Yang and Qiu [12], Golan et al. [15], Kumar et al. [16] and Soofi [17]. It is founded on the fact that the decision maker wishes less uncertainty and bigger expected utility.

Like the EU-E measure of risk, if all actions have an equal normalized expected utility, then the risk ordering is determined by the normalized entropy component. If all states of nature have the same distribution, then the normalized entropy of the states is the same for every state of nature corresponding to each action; in this case the risk ordering is determined by the normalized expected utility component.

This measure of risk builds on the basis that people's perception of risk depends on two factors: relative uncertainty of the outcomes and expected utility of risky actions. For some people, relative uncertainty far outweighs expected utility and for other people the expected utility outweighs the uncertainty. In the evaluation of the risk, people may distinguish relative uncertainty and expected utility by tradeoff coefficient $\lambda$.

It should be noted that both the EU-E and normalized EU-E measures of risk are linear weighted averages of the normalized expected utility and (normalized) entropy. Expected utility and entropy alone are not the measure of risk. It may not be true if someone only takes expected utility and entropy alone as a measure of risk.

We assume the individual makes decisions according to the normalized EU-E measure of risk. This gives the following definition of the normalized EU-E decision model.

Definition 2.2. For a given general decision analysis model $G=(\Theta, A, U)$, action $a_{1}, a_{2} \in A$, $R\left(a_{1}\right), R\left(a_{2}\right)$ denote normalized EU-E measure of risk of $a_{1}$ and $a_{2}$ respectively. If $R\left(a_{1}\right)<R\left(a_{2}\right)$, then action $a_{1}$ is preferred to action $a_{2}$ in the sense of normalized EU-E measure of risk, denoted by $a_{1} \succ a_{2}$, or $a_{2} \prec a_{1}$; if $R\left(a_{1}\right) \leq R\left(a_{2}\right)$, then action $a_{2}$ is not superior to $a_{1}$, denoted by $a_{1} \succeq a_{2}$.

According to Definition 2.2, we can rank the actions in action space by the order of normalized EU-E measure of risk. Among all actions in action space, the one with the minimal normalized EU-E risk value is optimal.

\section{Properties of the Normalized EU-E Measure of Risk}

In this section, we discuss some normative properties of the normalized expected utility and entropy measure of risk. We can obtain the following results directly from Definition 2.1:

Proposition 1. Given a general decision analysis model $G=(\Theta, A, u), a_{1}, a_{2} \in A$, the normalized entropy of the state $\theta$ corresponding to action $a_{i}$ is denoted by $N H_{a_{1}}(\theta)(i=1,2)$. Denote expected utility of $a_{i}$ by $E\left(a_{i}\right)$, i.e., $E\left(a_{i}\right)=E\left[u\left(X\left(a_{i}, \theta\right)\right)\right](i=1,2)$. There holds the following results.

(1) If $E\left(a_{1}\right)=E\left(a_{2}\right)$, and $N H_{a_{1}}(\theta)<N H_{a_{2}}(\theta)$, then $R\left(a_{1}\right)<R\left(a_{2}\right)$. 
(2) If $N H_{a_{1}}(\theta)=N H_{a_{2}}(\theta)$, and $E\left(a_{1}\right)>E\left(a_{2}\right)$, then $R\left(a_{1}\right)<R\left(a_{2}\right)$.

(3) If $N H_{a_{1}}(\theta)<N H_{a_{2}}(\theta)$, and $E\left(a_{1}\right)>E\left(a_{2}\right)$, then $R\left(a_{1}\right)<R\left(a_{2}\right)$.

By Proposition 1, we know that for two different actions in the action space with the same expected utility, the action with less normalized entropy has smaller risk. We also know that the riskiness of an action with higher expected utility is less when normalized entropies of their corresponding states of nature are equal. This is consistent with people's perception of risk.

Proposition 2. Given a general decision analysis model $G=(\Theta, A, u)$, we have:

(1) If expected utilities of all actions in action space $A$ are the same, then the action with the least normalized entropy is the optimal one.

(2) If the normalized entropies of the states corresponding to each action in action space $A$ are equal, then the action with the largest expected utility is optimal.

This can directly follow from Definition 2.1.

By Proposition 2, if all actions in the action space have the same expected utility, we only need to compare their normalized entropies when making a decision. The action with the smallest normalized entropy is the optimal one. If all actions have the same normalized entropies, we only need to compare their expected utilities, and the action with the largest expected utility is the optimal one. In this case, we take $\lambda=0$, we choose the action with less normalized EU-E measure of risk as the preferred one, that is, the action with higher expected utility. Thus, the normalized EU-E decision criterion is consistent with the expected utility principle.

Specifically, if one of actions $a_{1}$ and $a_{2}$ is a certain action, i.e., the occurrence of one outcome is certain with probability 1 , the other is a risky one, then we have the following proposition.

Proposition 3. Given a general decision analysis model $G=(\Theta, A, u)$, suppose the decision maker is risk averse $\left(u(x) \geq 0, u^{\prime}(x) \geq 0, u^{\prime \prime}(x) \leq 0\right), A=\left\{a_{1}, a_{2}\right\} . a_{1}$ is the action with a certain outcome $c$, and $a_{2}=\left(x_{1}, p_{1} ; x_{2}, p_{2} ; \cdots ; x_{n}, p_{n}\right)$ (assuming at least one of $p_{1}, p_{2}, \ldots, p_{n}$ less than 1$)$. If action $a_{1}$ and $a_{2}$ have the same expected value, then $a_{1}$ is the preferred action in the sense of the normalized EU-E model.

Proof: Since action $a_{1}$ and $a_{2}$ have the same expected value, then we have $\sum_{i=1}^{n} p_{i} x_{i}=c$. Furthermore, the decision maker is risk averse, so the utility function is concave. By Jensen inequality, we have $u\left(\sum_{i=1}^{n} p_{i} x_{i}\right) \geq \sum_{i=1}^{n} p_{i} u\left(x_{i}\right)$. Thus, $u(c) \geq \sum_{i=1}^{n} p_{i} u\left(x_{i}\right)$.

Therefore, we have $E\left(a_{1}\right)=u(c) \geq E\left(a_{2}\right)=\sum_{i=1}^{n} p_{i} u\left(x_{i}\right)$. Thus, $\max _{a \in A}\{E[u(X(a, \theta))]\}=u(c)$.

Thus, for any tradeoff coefficient $\lambda(0 \leq \lambda \leq 1)$, the normalized EU-E measures of risk of action $a_{1}$ and $a_{2}$ are as follows:

$$
R\left(a_{1}\right)=-(1-\lambda), R\left(a_{2}\right)=\lambda N H_{a_{2}}(\theta)-(1-\lambda) \sum_{i=1}^{n} p_{i} u\left(x_{i}\right) / u(c) .
$$

Obviously, it holds that $R\left(a_{1}\right)<R\left(a_{2}\right)$. Thus, action $a_{1}$ is the preferred action in the sense of the normalized EU-E model. 
Proposition 3 can explain the phenomenon why people choose the action with certain outcomes rather than risky actions when these actions have same expected value.

For the above specific problem, if we make decisions by the expected utility criterion, we can also obtain that action $a_{1}$ is optimal. That is, for this decision problem, we can get the same decision choice both by the normalized EU-E model and the expected utility principle. A risk averter can get the conclusion by intuition, so the results are consistent made both by intuition and the normalized EU-E model. By this proposition, we can show the normalized EU-E model can serve either as a descriptive model or a normative decision model.

Similar to EU-E measure of risk, the following proposition shows that the normalized EU-E measure of risk fits empirical findings concerning people's perception of risk quite well.

Proposition 4. Given a general decision analysis model $G=\{\Theta, A, u\}$ with nonnegative outcomes, there hold the following results.

(1) If $A=\{a, \alpha+a\}$, where $\alpha>0$ is a constant, then:

$$
R(a+\alpha)<R(a)
$$

namely, risk decreases when a positive constant is added to all outcomes of an action.

(2) If $A=\{a, \beta a\}$, where $\beta>1$ is a constant, then:

$$
R(\beta a)<R(a),
$$

i.e., risk decreases if a constant greater than 1 multiplies all outcomes of an action, that is risk decreases with an increase in range of the outcomes of risky action.

Proof. (1) Since utility function $u(X)$ is increasing, then for $\alpha>0$, we have:

$$
u(X+\alpha)>u(X)
$$

Then:

$$
\max _{a \in A}\{E[u(X(a, \theta))]\}=\max \{E[u(X)], E[u(X+\alpha)]\}=E[u(X+\alpha)] .
$$

Thus, by Definition 2.1, we have:

$$
\begin{gathered}
R(a)=\lambda H_{a}(\theta) / \ln (n)-(1-\lambda) E[u(X(a, \theta))] / \max _{a \in A}\{E[u(X(a, \theta))]\} \\
=\lambda H_{a}(\theta) / \ln (n)-(1-\lambda) E[u(X)] / E[u(X+\alpha)], \\
R(a+\alpha)=\lambda H_{a+\alpha}(\theta) / \ln (n)-(1-\lambda) .
\end{gathered}
$$

For action $a$ and $a+\alpha, H_{a}(\theta)=H_{a+\alpha}(\theta)$, and $\ln (n)$ is a constant. By Equations (10) and (11), we can reach the desired conclusion.

(2) Since utility function is increasing and outcomes $X=X(a, \theta)$ are all nonnegative, then we have $u(\beta X)>u(X)$ for $\beta>1$. Moreover $A=\{a, \beta a\}$, thus:

$$
\max _{a \in A}\{E[u(X(a, \theta))]\}=\max \{E[u(X)], E[u(\beta X)]\}=E[u(\beta X)] .
$$

Therefore:

$$
R(a)=\lambda H_{a}(\theta) / \ln (n)-(1-\lambda) E[u(X)] / E[u(\beta X)]
$$




$$
R(\beta a)=\lambda H_{\beta a}(\theta) / \ln (n)-(1-\lambda)
$$

For action $a$ and $\beta a, H_{a}(\theta) / \ln (n)=H_{\beta a}(\theta) / \ln (n)$, thus we have $R(\beta a)<R(a)$.

\section{Explanations of the Certainty Effect}

Expected utility serves as a descriptive model for interpreting both economic behavior and human decision behavior to some extent, but it has been challenged by several anomalies and empirical studies [2,18,19]. Using EU-E model and EM-EU representation of gambles [11], the Allais paradox ([18]) can be interpreted in a simple as well as reasonable way.

In expected utility theory, the utilities of outcomes are weighted by their probabilities. Kahneman and Tversky [2] have described a series of risky choices in which people's preferences systematically violated this principle. They have shown that people often overweight outcomes that are considered certain, relative to outcomes which are merely probable-a phenomenon which they called the certainty effect. Now, the certainty effects are the two special cases of general empirical phenomena called the common ratio effect and the common consequence effect, respectively [20]. Using the normalized EU-E model, the certainty effect in prospect theory ([2]) can be interpreted in a reasonable way. It demonstrates the reasonability of the normalized EU-E model as a descriptive model.

\subsection{Explanations of a Case of Common Ratio Effect}

The common ratio effect comes from Machina [20]. It is a phenomenon involving pairs of prospects of the following form:

$$
a_{1}=\left(\begin{array}{cc}
x & 0 \\
p & 1-p
\end{array}\right) \text { versus } a_{2}=\left(\begin{array}{cc}
y & 0 \\
q & 1-q
\end{array}\right)
$$

and:

$$
a_{3}=\left(\begin{array}{cc}
x & 0 \\
r p & 1-r p
\end{array}\right) \text { versus } a_{4}=\left(\begin{array}{cc}
y & 0 \\
r q & 1-r q
\end{array}\right)
$$

where $0<x<y, 1 \geq p>q>0$ ( or $x>y>0,0<p<q \leq 1$ ), $0<r<1$.

One kind of certainty effect of Kahneman and Tversky [2] is included as a special case of the common ratio effect [20]. The expected utility model predicts choices of $a_{1}$ and $a_{3}$ or else $a_{2}$ and $a_{4}$. However, experimental studies have found a systematic tendency for choices to depart from these predictions [20].

Marley and Luce [7] studied these similar risky choices and gave the definition of common ratio independence as follows:

Common ratio independence is satisfied if, for all $0 \leq p, q \leq 1,0<r \leq 1 / \max (p, q)$, $a_{1}=\left(\begin{array}{cc}x & 0 \\ p & 1-p\end{array}\right) \succsim a_{2}=\left(\begin{array}{cc}y & 0 \\ q & 1-q\end{array}\right)$ is equivalent to $a_{3}=\left(\begin{array}{cc}x & 0 \\ r p & 1-r p\end{array}\right) \succsim a_{4}=\left(\begin{array}{cc}y & 0 \\ r q & 1-r q\end{array}\right)$. The common ratio effect is just the contrary of the common ratio independence. 
Let $x=6000, p=0.45, y=3000, q=0.9, r=1 / 450$, then this special case of the common ratio effect is the example of this kind of certainty effect in Kahneman and Tversky [2] involving only two outcome lotteries in the following pairs of risky actions.

Problem 1. Select $a_{1}$ or $a_{2}$, where:

$$
a_{1}=\left(\begin{array}{cc}
6000 & 0 \\
0.45 & 0.55
\end{array}\right), a_{2}=\left(\begin{array}{cc}
3000 & 0 \\
0.90 & 0.10
\end{array}\right) .
$$

Problem 2. Choose $a_{3}$ or $a_{4}$, where:

$$
a_{3}=\left(\begin{array}{cc}
6000 & 0 \\
0.001 & 0.999
\end{array}\right), a_{4}=\left(\begin{array}{cc}
3000 & 0 \\
0.002 & 0.998
\end{array}\right) .
$$

The above problems can be summarized using the general decision making model in Table 2 as follows.

Table 2. Example of common ratio effect.

\begin{tabular}{cccccc}
\hline $\begin{array}{c}\text { Risky } \\
\text { Choice }\end{array}$ & $\begin{array}{c}\text { Outcomes and Their } \\
\text { Corresponding Probabilities }\end{array}$ & $\begin{array}{c}\text { Expected } \\
\text { Value }\end{array}$ & Entropy & $\begin{array}{c}\text { Normalized } \\
\text { Entropy }\end{array}$ \\
\hline$a_{1}$ & 6000 & 0 & 2700 & 0.6881 & 0.9928 \\
0.45 & 0.55 & 0 & 2700 & 0.3251 & 0.4690 \\
\hline$a_{2}$ & 3000 & 0.10 & 6 & 0.0079 & 0.0114 \\
\hline$a_{3}$ & 0.90 & 0 & & & \\
\hline & 0.001 & 0.999 & 0 & 0.0144 & 0.0208 \\
\hline
\end{tabular}

Empirical studies have shown that a majority of people have the preference pattern of $a_{2}$ over $a_{1}$, but $a_{3}$ over $a_{4}$, which violates the expected utility principle [2]. The phenomenon illustrates a situation in which the most people's attitudes toward risk cannot be captured by the expected utility model.

We now use the normalized EU-U model to give a reasonable explanation of the above risky choices.

The expected values and normalized entropies of these four risky actions are shown in Table 2 as well. Since:

$$
E\left(a_{1}\right)=E\left(a_{2}\right)=2700, N H_{a_{1}}>N H_{a_{2}} ; E\left(a_{3}\right)=E\left(a_{4}\right)=6, N H_{a_{3}}<N H_{a_{4}} ;
$$

namely, actions $a_{1}$ and $a_{2}$ have the same expected value, but action $a_{2}$ has less normalized entropy, i.e., less measure of relative uncertainty. If the decision maker is risk averse, the by proposition 1, we conclude that action $a_{2}$ is superior to action $a_{1}$. Similarly, the action $a_{3}$ is superior to $a_{4}$.

In this example, the numbers of states of nature corresponding to each action are equal. Thus, it makes no difference using either the EU-E or the normalized EU-E model. So, if we use the EU-E model to give an explanation of the above risky action, it is the same as the normalized EU-E model. Now, we compare the normalized EU-E representation with EM-EU representation in Luce et al. [11].

From Table 2, we have: 


$$
\begin{gathered}
U\left(a_{1}\right)=E\left[u\left(a_{1}\right)\right]+A \cdot H_{a_{1}}(\theta)=2700+0.688 A, \\
U\left(a_{2}\right)=E\left[u\left(a_{2}\right)\right]+A \cdot H_{a_{2}}(\theta)=2700+0.325 A .
\end{gathered}
$$

Thus, $a_{2} \succ a_{1} \Leftrightarrow U\left(a_{1}\right)<U\left(a_{2}\right) \Leftrightarrow 2700+0.668 A<2700+0.325 A \Leftrightarrow A<0$.

And, $U\left(a_{3}\right)=E\left[u\left(a_{3}\right)\right]+A \cdot H_{a_{3}}(\theta)=6+0.008 A, U\left(a_{4}\right)=E\left[u\left(a_{4}\right)\right]+A \cdot H_{a_{4}}(\theta)=6+0.014 A$. So, we have $a_{3} \succ a_{4} \Leftrightarrow U\left(a_{3}\right)>U\left(a_{4}\right) \Leftrightarrow 0.008 A>0.014 A \Leftrightarrow A<0$.

In both cases, it mean the uncertainty of the state of nature will reduce the "total" utility of risky actions, i.e., the individual will choose the risky choices with less uncertainty when the risky choices have equal expect utilities. In either of these cases, the constant $A$ is less than 0 . This explanation is consistent with that of (normalized) EU-E representation for risky choices. Furthermore, this demonstrates the result (1) of Proposition 1.

If we let $x=3000, p=1, y=4000, q=0.8, r=0.25$, then this special case of the common ratio effect is another situation of certainty effect in Kahneman and Tversky [2], which involves choosing between pairs of two outcome lotteries. This case of the common ratio effect can be summarized using general decision-making model directly in Table 3.

\begin{tabular}{|c|c|c|c|c|c|}
\hline $\begin{array}{l}\text { Risky } \\
\text { Choice }\end{array}$ & $\begin{array}{r}\text { Outcon } \\
\text { Correspong }\end{array}$ & $\begin{array}{l}\text { heir } \\
\text { abilities }\end{array}$ & $\begin{array}{c}\text { Expected } \\
\text { Value }\end{array}$ & Entropy & $\begin{array}{c}\text { Normalized } \\
\text { Entropy }\end{array}$ \\
\hline$a_{1}$ & & & 3000 & 0 & 0 \\
\hline$a_{2}$ & $\begin{array}{l}4000 \\
0.80 \\
\end{array}$ & $\begin{array}{c}0 \\
0.20 \\
\end{array}$ & 3200 & 0.50 & 0.72 \\
\hline$a_{3}$ & $\begin{array}{l}3000 \\
0.25 \\
\end{array}$ & $\begin{array}{c}0 \\
0.75 \\
\end{array}$ & 750 & 0.56 & 0.81 \\
\hline$a_{4}$ & $\begin{array}{l}4000 \\
0.20\end{array}$ & $\begin{array}{c}0 \\
0.80\end{array}$ & 800 & 0.50 & 0.72 \\
\hline
\end{tabular}

Table 3. Example of common ratio effect.

Experiments have shown that a majority of subjects have the preference pattern of $a_{1}$ over $a_{2}$ but $a_{4}$ over $a_{3}$ [2]. The modal pattern preferences in this case are not compatible with expected utility theory.

If the decision maker is risk neutral, i.e., the utility function is linear, and $u(x)=x$, then the normalized EU-E measure of risk of action $a_{1}$ and $a_{2}$ are as follows:

$$
R\left(a_{1}\right)=-0.94(1-\lambda), R\left(a_{2}\right)=0.72 \lambda-(1-\lambda)=1.72 \lambda-1 .
$$

By the normalized EU-E decision model, the sufficient and necessary condition for $a_{1} \succ a_{2}$ is $-0.94(1-\lambda)<1.72 \lambda-1$. So, we have $0.08<\lambda \leq 1$. Namely, when the tradeoff coefficient $0.08<\lambda \leq 1$, then we can predict the subject's preference pattern.

For action $a_{3}$ and $a_{4}$, since $E\left(a_{3}\right)<E\left(a_{4}\right)$, and $N H_{a_{3}}>N H_{a_{4}}$, then for any $0 \leq \lambda \leq 1$, we have $R\left(a_{3}\right)>R\left(a_{4}\right)$. Thus, we can reach the conclusion that action $a_{4}$ is superior to $a_{3}$. 
If the decision maker's utility function is $u(x)=\sqrt{x}$, the expected utilities of action $a_{1}$ and $a_{2}$ are as follows, respectively: $E\left(a_{1}\right)=54.77, E\left(a_{2}\right)=50.60$. We have $E\left(a_{1}\right)>E\left(a_{2}\right)$ and $N H_{a_{1}}<N H_{a_{2}}$, so action $a_{1}$ is superior to $a_{2}$.

For action $a_{3}$ and $a_{4}$, we have $E\left(a_{3}\right)=13.69, E\left(a_{4}\right)=12.65$. Their normalized EU-E measures of risk are as follows, respectively:

$$
R\left(a_{3}\right)=1.81 \lambda-1, R\left(a_{4}\right)=1.64 \lambda-0.92 .
$$

Then, we have $R\left(a_{3}\right)>R\left(a_{4}\right) \Leftrightarrow 0.47<\lambda \leq 1$. Thus, when $0.47<\lambda \leq 1$, the subjects will choose action $a_{4}$.

For the above explanations of these problems we can know that the decision maker's risky choices are compatible with the normalized EU-E decision model as long as the tradeoff coefficient $\lambda$ is rather big to some extent.

Similar to the above situation, the numbers of states of nature corresponding to each state of nature are equal or very close to each other. Thus, if we use the EU-E model to explain the above risky action, it is similar to the normalized EU-E model.

If the decision maker is risk neutral, using the EU-E decision model, when the tradeoff coefficient $0.08<\lambda \leq 1$, then we can predict the subject's preference pattern.

For action $a_{3}$ and $a_{4}$, then for any $0 \leq \lambda \leq 1$, we have $R\left(a_{3}\right)>R\left(a_{4}\right)$. Thus, we can reach the conclusion that action $a_{4}$ is superior to $a_{3}$.

We make a comparison with the EM-EU representation in Luce et al. [11]. From Table 3, we have:

$$
U\left(a_{1}\right)=3000, U\left(a_{2}\right)=3200+0.50 A .
$$

Thus, $a_{1} \succ a_{2} \Leftrightarrow U\left(a_{1}\right)>U\left(a_{2}\right) \Leftrightarrow 3000>3200+0.50 A \Leftrightarrow A<-400$.

Moreover, $U\left(a_{3}\right)=750+0.56 \mathrm{~A}, U\left(a_{4}\right)=800+0.50 \mathrm{~A}$, so we have:

$$
a_{3} \prec a_{4} \Leftrightarrow U\left(a_{3}\right)<U\left(a_{4}\right) \Leftrightarrow 750+0.56 A<800+0.50 A \Leftrightarrow A<833.33 .
$$

In these two cases, EM-EU representation for risky actions may not provide an intuitive explanation for empirical results. Even though a value of $A<-400$ explains the two pairs of choices, the meaning of constant $A$ may not be clear to these examples. One might think more about why the estimate of $A$ is so different for the two pairs of gambles.

\subsection{Explanations of a Case of Common Consequence Effect}

The common consequence effect comes from Machina [20] as well. It is a general phenomenon involving pairs of probability mixturing actions in the following form:

$$
a_{1}: \alpha \delta_{x}+(1-\alpha) P^{* *} \text { versus } a_{2}: \alpha P+(1-\alpha) P^{* *}
$$

and:

$$
a_{3}: \alpha \delta_{x}+(1-\alpha) P^{*} \text { versus } a_{4}: \alpha P+(1-\alpha) P^{*}
$$

where $\delta_{x}$ denotes the prospect which yields $x$ with certainty, $P$ involves outcomes both greater and less than $x$, and $P^{* *}$ stochastically dominates $P^{*}$. Many experiments have shown that a majority of 
subjects have the preference pattern of $a_{1}$ over $a_{2}$ in the first pair, and $a_{4}$ over $a_{3}$ in the second, which is inconsistent with any form of expected utility $([2,21])$.

Let $\delta_{x}=\left(\begin{array}{c}2400 \\ 1\end{array}\right), \alpha=0.34, P=\left(\begin{array}{cc}2500 & 0 \\ 33 / 34 & 1 / 34\end{array}\right), P^{*}=\left(\begin{array}{l}0 \\ 1\end{array}\right), P^{* *}=\left(\begin{array}{c}2400 \\ 1\end{array}\right)$, there holds $P^{* *}$ stochastically dominates $P^{*}$. Then, we have the following results:

$$
\begin{aligned}
& a_{1}: \alpha \delta_{x}+(1-\alpha) P^{* *}=0.34\left(\begin{array}{c}
2400 \\
1
\end{array}\right)+0.66\left(\begin{array}{c}
2400 \\
1
\end{array}\right)=\left(\begin{array}{c}
2400 \\
1
\end{array}\right), \\
& a_{2}: \alpha P+(1-\alpha) P^{* *}=0.34\left(\begin{array}{cc}
2500 & 0 \\
33 / 34 & 1 / 34
\end{array}\right)+0.66\left(\begin{array}{c}
2400 \\
1
\end{array}\right)=\left(\begin{array}{ccc}
2500 & 2400 & 0 \\
0.33 & 0.66 & 0.01
\end{array}\right) \text {, } \\
& a_{3}: \alpha \delta_{x}+(1-\alpha) P^{*}=0.34\left(\begin{array}{c}
2400 \\
1
\end{array}\right)+0.66\left(\begin{array}{l}
0 \\
1
\end{array}\right)=\left(\begin{array}{cc}
2400 & 0 \\
0.34 & 0.66
\end{array}\right) \text {, } \\
& a_{4}: \alpha P+(1-\alpha) P^{*}=0.34\left(\begin{array}{cc}
2500 & 0 \\
33 / 34 & 1 / 34
\end{array}\right)+0.66\left(\begin{array}{l}
0 \\
1
\end{array}\right)=\left(\begin{array}{cc}
2500 & 0 \\
0.33 & 0.67
\end{array}\right) \text {. }
\end{aligned}
$$

This special case of the common consequence effect is another kind of certainty effect in Kahneman

\begin{tabular}{|c|c|c|c|c|c|c|}
\hline $\begin{array}{l}\text { Risky } \\
\text { Choice }\end{array}$ & \multicolumn{3}{|c|}{$\begin{array}{l}\text { Outcomes and Their } \\
\text { Corresponding Probabilities }\end{array}$} & $\begin{array}{c}\text { Expected } \\
\text { Value }\end{array}$ & Entropy & $\begin{array}{c}\text { Normalized } \\
\text { Entropy }\end{array}$ \\
\hline$a_{1}$ & \multicolumn{3}{|c|}{2400} & 2400 & 0 & 0 \\
\hline$a_{2}$ & $\begin{array}{c}2500 \\
0.33 \\
\end{array}$ & $\begin{array}{c}2400 \\
0.66\end{array}$ & $\begin{array}{c}0 \\
0.01 \\
\end{array}$ & 2409 & 0.686 & 0.62 \\
\hline$a_{3}$ & $\begin{array}{c}2400 \\
0.34\end{array}$ & & $\begin{array}{l}0 \\
0.66\end{array}$ & 816 & 0.641 & 0.92 \\
\hline$a_{4}$ & $\begin{array}{l}2500 \\
0.33\end{array}$ & & $\begin{array}{l}0 \\
0.67\end{array}$ & 825 & 0.634 & 0.91 \\
\hline
\end{tabular}
and Tversky's prospect theory [2], which can be summarized using the general decision model in Table 4 as follows.

Table 4. Example of common consequence effect.

Suppose the decision maker is risk neutral, and the utility function is $u(x)=x$, then for actions $a_{1}$ and $a_{2}$, their normalized EU-U measures of risk are as follows respectively:

$$
R\left(a_{1}\right)=-0.996(1-\lambda), R\left(a_{2}\right)=1.62 \lambda-1 .
$$

As long as the tradeoff factor $0.006<\lambda \leq 1$, then we have $R\left(a_{1}\right)<R\left(a_{2}\right)$, which predicts the preference pattern. For actions $a_{3}$ and $a_{4}, E\left(a_{3}\right)<E\left(a_{4}\right)$ and $N H_{a_{3}}(\theta)>N H_{a_{4}}(\theta)$, so $a_{4}$ is superior to $a_{3}$ in which the preference is consistent with the normalized EU-U measure of risk.

We have interpreted this kind of certainty effect by the normalized EU-E model in a simple way. From the above interpretations, we know that the preference pattern fits with the normalized EU-E decision criterion. Thus, the normalized EU-E model shows the descriptive ability for risk behaviours and it serves as a descriptive decision model to some extent in this kind of situations. 
The situation is the same as that of the common ratio effect above. If we use the EU-E model to explain the above risky action, it is similar to the normalized EU-E model.

If the decision maker is risk neutral, using the EU-E decision model, when the tradeoff coefficient $0.006<\lambda \leq 1$, then we can predict the subject's preference pattern in the first pairs of choice.

For action $a_{3}$ and $a_{4}$, then for any $0 \leq \lambda \leq 1$, we have $R\left(a_{3}\right)>R\left(a_{4}\right)$. Thus, we can reach the conclusion that action $a_{4}$ is superior to $a_{3}$, in which the preference is consistent with the EU-U measure of risk.

We make a comparison with EM-EU representation in Luce et at. [11]. From Table 4, we have:

$$
U\left(a_{1}\right)=2400, U\left(a_{2}\right)=2409+0.686 \mathrm{~A} .
$$

Thus, $a_{1} \succ a_{2} \Leftrightarrow U\left(a_{1}\right)>U\left(a_{2}\right) \Leftrightarrow 2400>2409+0.686 A \Leftrightarrow A<-13.12$.

Moreover, $U\left(a_{3}\right)=816+0.641 A, U\left(a_{4}\right)=825+0.634 A$, so we have:

$$
a_{4} \succ a_{3} \Leftrightarrow U\left(a_{4}\right)>U\left(a_{3}\right) \Leftrightarrow 825+0.634 A>816+0.641 A \Leftrightarrow A<1285.7 .
$$

Similar to second example in Section 4.1, for these two risky choices, EM-EU representation may not provide an intuitive explanation for the empirical results. The meaning of constant $A$ may not be "sensible" in explaining the results. What is more, the EM-EU representation builds on the segregation assumption, so one concern here is that if there is enough empirical support for segregation assumption in Luce et al. [11].

\subsection{Further Discussion}

For the risky actions in Sections 4.1 and 4.2, we use the normalized EU-U decision model to give reasonable explanations for the certainty effect. Actually, we may use EU-U and EM-EU representations to predict the risky choices as well when the numbers of state of nature are equal, or relatively close. EU-U and EM-EU representations are not proper models to predict the risky choices when numbers of state of nature are far apart. In this case, we need to use the normalized EU-E model. In the introduction, we have discussed the risky choices between pairs of risky choices, in which numbers of state of nature are relatively apart. In this example, they have the same expected value 10 and normalized entropy. If the utility function is $u(x)=\sqrt{x}$, then $E\left(a_{1}\right)=3.161, E\left(a_{2}\right)=3.158$. Using the normalized EU-E measure of risk, we should choose the $a_{1}$. This is consistent with the normalized EU-E decision model.

\section{Conclusions}

In this paper, by combining normalized expected utility and entropy together, we propose the normalized EU-E measure of risk. The normalized EU-E measure of risk lies between -1 and 1 . It has some normative properties under certain conditions. In the case where the normalized entropies of all actions are equal, the normalized EU-E decision criterion is consistent with the expected utility principle. Moreover, it has the descriptive power to some extent. We also compare the predictions of the (normalized) EU-E and EM-EU presentations. When the numbers of state of nature are close, all these representation can be the descriptive models for risky choices, but when the numbers of state of nature are far apart, only the normalized EU-E is the proper descriptive model. The two kinds of 
certainty, which are the special case of common ratio and common consequences effect, can be interpreted reasonably using the normalized EU-E model.

\section{Acknowledgement}

The authors thank the three anonymous referees for their very careful and helpful comments and suggestions to make it possible to add color to this version of the paper. We thank one of the referee's careful comments and these useful comments helped us a lot to improve the paper. We also thank the other two referees for their very high evaluations of our paper and these praises stimulate us to further this study! Actually, one of the referees' comments suggest us the very good topics for further research, for example, if the use of other entropies, such as entropies of degree $\alpha$ and Renyi's entropy, will support the model; in addition, if there is an experimental design to estimate the coefficient $\lambda$ that fits an individual. The first author thanks the very early comments and suggestions that R. Duncan Luce has given to me when I visited University of California, Irvine from June to September, 2009. This paper may also be a memory of R. Duncan Luce. We also thank the financial support by the National Natural Science Foundation of China (Grant No. 71271011).

\section{Author Contributions}

Jiping Yang conceived, designed, and performed the study. Jiping Yang and Wanhua Qiu collected and analysed the examples in the paper. Jiping Yang and Wanhua Qiu wrote and revised the paper together. The authors have read and approved the final published manuscript.

\section{Conflicts of Interest}

The authors declare no conflict of interest.

\section{References}

1. Von Neumann, J.; Morgenstern, O. The Theory of Games and Economic Behavior; Princeton University Press: Princeton, NJ, USA, 1944.

2. Kahneman, D.; Tversky, A. Prospect theory: An analysis of decision under risk. Econometrica 1979, 47, 263-291.

3. Levy, H. The definition of risk: An extension. J. Econ. Theory 1977, 14, 232-234.

4. Dyer, J.S.; Jia, J. Relative risk-value models. Eur. J. Oper. Res. 1997, 103, 170-185.

5. Jia, J.; Dyer, J.S.; Butler, J.C. Measures of perceived risk. Manag. Sci. 1999, 45, 519-532.

6. Dionisio, A.; Reis, A.H.; Coelho, L. Utility function estimation: The entropy approach. Physica $A$ 2008, 387, 3862-3867.

7. Marley, A.A.J.; Luce, R.D. Independence properties vis-à-vis several utility representations. Risk. Decis. 2005, 58, 77-143.

8. Ng, C.T.; Luce, R.D.; Marley, A.A.J. On the utility of gambling: Extending the approach of Meginniss (1976). Aequationes Mathematicae 2008, 76, 281-304. 
9. Luce, R.D.; Marley, A.A.J.; Ng, C.T. Entropy-related measures of the utility of gambling. In The Mathematics of Preference, Choice, and Order: Essays in Honor of Peter C. Fishburn; Brams, S., Gehrlein, W., Roberts, F., Eds.; Springer: Berlin/Heidelberg, Germany, 2008; pp. 5-25.

10. Luce, R.D.; Ng, C.T.; Marley, A.A.J.; Aczél, J. Utility of gambling I: Entropy modified linear weighted utility. Econ. Theory 2008, 36, 1-33.

11. Luce, R.D.; Ng, C.T.; Marley, A.A.J.; Aczél, J. Utility of gambling II: Risk, paradoxes, and data. Econ. Theory 2008, 36, 165-187.

12. Yang, J.; Qiu, W. A measure of risk and a decision-making model based on expected utility and entropy. Eur. J. Oper. Res. 2005, 164, 792-799.

13. Luce, R.D. Several possible measures of risk. Risk. Decis. 1980, 12, 217-228; Correction, 1981, 13,381 .

14. Shannon, C.E. A mathematical theory of communication. Bell Syst. Tech. J. 1948, 27, 379-423.

15. Golan, A.; Judge, G.; Miller, D. Maximum Entropy Econometrics: Robust Estimation with Limited Data; Wiley: New York, NY, USA, 1996.

16. Kumar, U.; Kumar, V.; Kapur, J.N. Normalized measures of entropy. Int. J. Gen. Syst. 1986, 12, 55-69.

17. Soofi, E.S. A generalizable formulation of conditional logit with diagnostics. J. Am. Stat. Assoc. 1992, 87, 812-816.

18. Allais, M. Le comportement de l'homme rationnel devant le risqué: Critique des postulats et axiomes de l'ecole americaine. Econometrica 1953, 21, 503-546.

19. Savage, L.J. Foundations of Statistics; Wiley: New York, NY, USA, 1954.

20. Machina, M.J. Choice under uncertainty: Problems solved and unsolved. Econ. Perspect. 1987, 1, 121-154.

21. Chew, S.H.; Waller, W. Empirical tests of weighted utility theory. J. Math. Psychol. 1986, 50, $55-72$.

(C) 2014 by the authors; licensee MDPI, Basel, Switzerland. This article is an open access article distributed under the terms and conditions of the Creative Commons Attribution license (http://creativecommons.org/licenses/by/3.0/). 